\title{
Online Targetless End-to-End Camera-LIDAR Self-calibration
}

\author{
Balázs Nagy ${ }^{2,1}$ \\ Levente Kovács ${ }^{1}$ \\ balazs.nagy@sztaki.mta.hu \\ levente.kovacs@sztaki.mta.hu \\ Csaba Benedek ${ }^{1,2}$ \\ csaba.benedek@sztaki.mta.hu \\ ${ }^{1}$ Machine Perception Research Laboratory \\ Institute for Computer Science and Control (MTA SZTAKI) \\ H-1111 Kende u. 13-17 Budapest, Hungary \\ ${ }^{2}$ Pázmany Péter Catholic University, Faculty of Inf. Tech. and Bionics \\ Práter u. 50/A, Budapes
}

\begin{abstract}
In this paper we propose an end-to-end, automatic, online camera-LIDAR calibration approach, for application in self driving vehicle navigation. The main idea is to connect the image domain and the 3D space by generating point clouds from camera data while driving, using a structure from motion (SfM) pipeline, and use it as the basis for registration. As a core step of the algorithm we introduce an object level alignment to transform the generated and captured point clouds into a common coordinate system. Finally, we calculate the correspondences between the $2 D$ image domain and the $3 D$ LIDAR point clouds, to produce the registration. We evaluated the method in various different real life traffic scenarios.
\end{abstract}

\section{Introduction}

State-of-the-art autonomous driving systems [14], equipped with 3D LIDAR sensors and electro-optical cameras can achieve accurate and comprehensive environment perception. While real time LIDARs, such as Velodye's rotating multi-beam (RMB) sensors measure directly $3 \mathrm{D}$ geometric information with a relatively low spatial resolution, optical cameras provide us high resolution visual color and texture information enabling to also investigate small details in the scene.

Accurate LIDAR and camera calibration is essential for robust data fusion, which issues are therefore deeply studied in the literature. The existing calibration techniques can be grouped based on various aspects [9]: depending on the necessity of user interaction, prescribed specific environmental conditions, and operational requirements, one can distinguish semi- of fully automatic, target-based or targetless, and offline or online approaches (see Sec. 2). In this paper, we propose a novel targetless and fully automatic extrinsic calibration method between a camera and an RMB LIDAR mounted on a moving car. Our technique requires only to place and fix the sensors onto the ve-

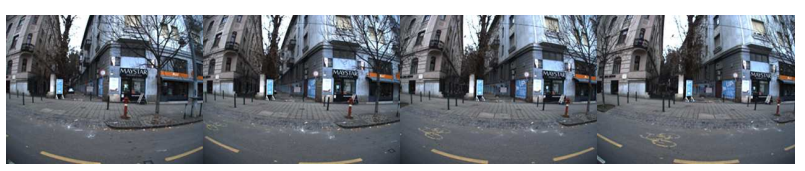

(a)

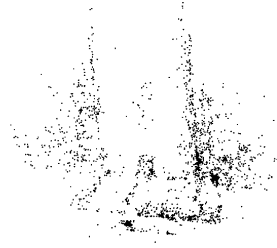

(b)

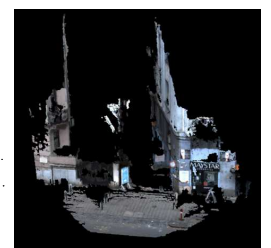

(c)
Figure 1. (a) 4 from a set of 8 images to process. (b) Generated sparse point cloud (2041 points).

(c) Densified point cloud (257796 points).

hicle top and to start driving in a typical dense urban environment, meanwhile the method calculates all the necessary registration parameters online. Considering that the RMB LIDAR point clouds are notably sparse and their density rapidly decreases as a function of the distance from the sensor, we only rely on robustly observable landmark objects, which are matched with blob components extracted from a synthesized 3D environment model obtained from the consecutive camera images by a Structure from Motion (SfM) approach.

The outline of the paper is as follows: A detailed literature overview of camera-LIDAR calibration is given in Sec. 2, while the steps of the proposed method are described in Sec. 3. In Sec. 4, we evaluate the new targetless and fully automatic method in real urban environment, and quantitatively demonstrate that its performance is close to a state-of-the-art target-based and semi-automatic calibration technique [11]. 


\section{Related works}

Target based methods use special calibration targets with a preliminary known size, such as polygonal planar boards [10], boxes [11], checkerboard patterns [3] or a simple printed circle [4]. Some of these techniques are semi-automatic, i.e. they require to take several images while the calibration pattern is manually moved [11], which approach may yield a notable accurate registration, but the calibration process is time consuming, and the quality depends on the operator's skills. Other methods attempt to automatically detect and match features on static targets: the approaches of $[4,13]$ are based on an automatic ellipse detector, $[16]$ detects holes on planar object, [3] uses one LIDARimage pair with multiple checkerboards adopting an improved corner detector, and [10] uses white homogeneous target objects to calibrate LIDAR and camera frames. While these approaches avoid user intervention during the calibration process, they can only be adopted in scenes where the calibration targets are available, and in some cases they need a complex installation of several carefully placed targets in a garage (e.g. [3] uses 12 checkerboards).

On the other hand, targetless approaches extract features for correspondence calculation directly from the observed natural environment without any calibration object. [15] transform the range sensor's 3D measurement into a new image, called Bearing Angle image (BA), and identifies point correspondences between the $\mathrm{BA}$ and the camera image via conventional image processing operations. Alternatively, mutual Information was introduced in [17] to calibrate different range sensors with cameras. However, experiments show that the above techniques require a critical point density of the point cloud for reliable operation, which is not ensured at the single RMB LIDAR frames provided by a car during self-driving operation [17]. The correspondences in [6] are detected based on automatically extracted sets of lines both in the 2D images and in the $3 \mathrm{D}$ point clouds. However, according to [6] the method is preferably adopted in indoor environment, where the required number of line correspondences can be often observed, which condition cannot be guaranteed in the sparse RMB LIDAR frames recorded in outdoor urban environment.

Even a well calibrated system needs some recalibration due to vibration on the roads and some sensor artifacts, so to avoid complex re-calibration offline process we propose an automatic, targetless, online registration method which is able to precisely calibrate LIDAR and camera sensors on the fly.

\section{The proposed approach}

Since state-of-the-art autonomous driving LIDAR sensors (e.g., Velodyne HDL64, VLP32 and VLP16) provide inhomogeneous, colorless, sparse point cloud

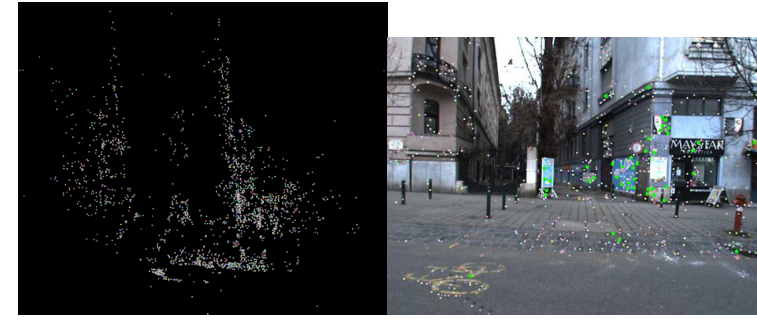

(a)

(b)

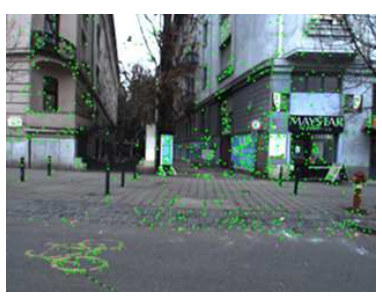

(c)

Figure 2. (a) Sparse cloud with each point assigned a unique color. (b) One frame showing color coded $2 \mathrm{D}$ points that contribute to the $3 \mathrm{D}$ point with the same color in (a). (c) Point cloud points reprojected to one of the respective $2 \mathrm{D}$ frames using the calculated transformation.

streams with typical patterns, the main bottleneck of online, targetless calibration approaches is the extraction of meaningful feature correspondences from the $3 \mathrm{D}$ point cloud and the $2 \mathrm{D}$ image domain.

To avoid feature (2/3D interest points, line and planar segments) detection from very different domains we turn to a structure from motion (SfM) based approach [7] to generate point clouds from the image sequences recorded by the moving vehicle, and we perform an object level alignment between the LIDAR and the generated point clouds. During the SfM process we calculate the transformation $T_{1}$ which projects the points of the generated point cloud onto the corresponding image pixels. Then we align the point clouds on the object level [8] and we estimate a transformation $T_{2}$ which transforms the LIDAR point cloud to the coordinate system of the generated one. At this stage we can project the points of the LIDAR point cloud directly onto the image domain using transformation $T_{1}$ and we save the mapping between the corresponding $3 \mathrm{D}$ points and the $2 \mathrm{D}$ pixel coordinates. Finally, using $T_{2}$ inverse we transform back the LIDAR point cloud to the original position and using the saved 2D$3 \mathrm{D}$ mapping information we calculate transformation $T_{3}$ which is able to project the original LIDAR point cloud directly onto the 2D image domain. 


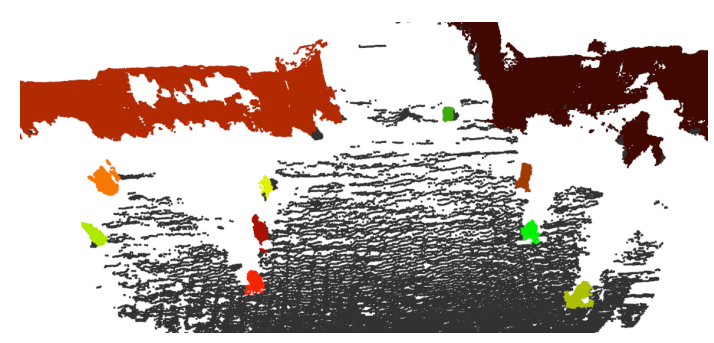

(a) Object detection result on densified SfM based point cloud

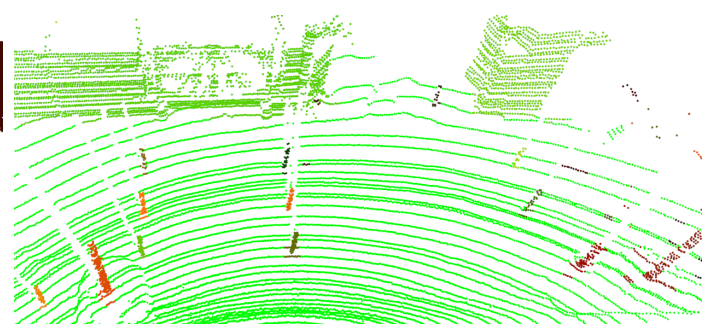

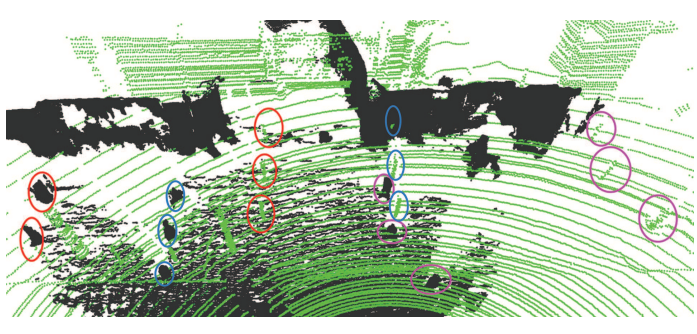

(c) Initial translation (b) Object detection result on Velodyne HDL64 LIDAR data

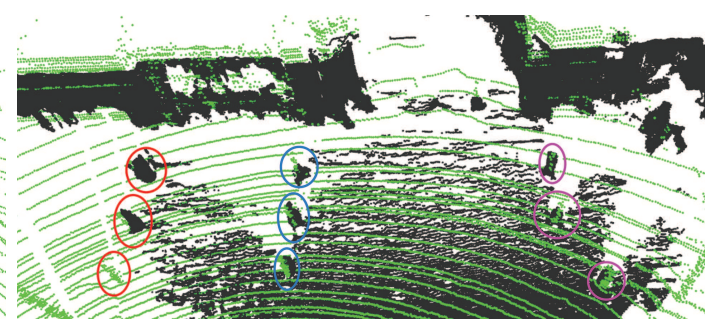

(d) Proposed approach

Figure 3. Results of the proposed object based alignment method. (c-d) Velodyne LIDAR data is displayed with green, while the generated point cloud is shown with dark grey. Identical color circles show the corresponding parts of the point clouds.

\subsection{Point cloud and transformation calculation from images}

The first step of the proposed method is to generate a point cloud from a finite, continuous window of camera images that can be used for alignment and registration. First, we genarate a sparse point cloud by taking consecutive camera images. As a basis for these calculations we use the OpenMVG ${ }^{1}$ library [7], with some modifications, as described in the following.

To obtain a set of images for producing a sparse point cloud, we start capturing image frames. We select a set of $N(N \geq 3)$ images $-N=8$ is used for the rest of the paper. The resolution of the camera we used was $1288 \times 964$ pixels. When selecting the frames, we specify a global motion threshold of $t h_{\text {move }} \geq 10$ pixels between consecutive frames, and feed these images into our structure from motion calculation pipeline, which has the following steps:

1) Image rectification: After selecting the $N$ frames (Fig. 1(a)), perform rectification and store the selected frames.

2) Extraction and matching $\left(L_{2}\right.$ fast cascade matching) of SIFT feature points for the selected images.

3) Structure from motion calculation: Perform the SfM, generate sparse point cloud (Fig. 1(b)) and store those image feature points that contribute to the sparse point cloud calculation with unique IDs. In this step, for each generated sparse $3 \mathrm{D}$ point we store all the $2 \mathrm{D}$ image points from all images that contributed to

\footnotetext{
${ }^{1}$ Url: https://github.com/openMVG/openMVG
}

the estimation of the current 3D point of the sparse point cloud. During the point cloud generation process we assign unique IDs to all 3D points and save their associated image points from all selected images.

4) Using the stored $3 \mathrm{D}-2 \mathrm{D}$ point associations for all processed frames (Fig. 2(a-b)), we select $M$ points from each processed frame based on point density (we used a constant $M=45$ ) and from these 2D-3D associations we calculate the transformation using solvePnP from OpenCV ${ }^{2}$. Then, to check that the transformation is usable, we reproject all the points to all images using the obtained transformation, and Fig. 2 (c) shows an example. In the case of this example the reprojection resulted in a very low average of 2.81 pixel reprojection error (averaged over all $N=8$ processed images).

5) Densification of the generated point cloud (Fig. 1(c)): For densification we use the OpenMVS ${ }^{3}$ library without modifications. The densified point cloud (Fig: $3(\mathrm{a})$ ) and the calculated transformation will be used in the next steps for the cloud alignment and registration.

The above calculations can be performed either continuously, by selecting the next $N$ frames in a moving time window, and updating the obtained transformations, or from time to time (e.g., every 5-10 minutes) since the vehicle's movements can cause slight (or not so slight) sensor displacements which can require regular updates. One benefit of the proposed approach is that there would be no need for returning to base

\footnotetext{
${ }^{2}$ Url: https://opencv.org/

${ }^{3} \mathrm{Url}$ : http://cdcseacave.github.io/openMVS
} 
to perform sensor calibrations, since the above calculations can be performed in situ, during the data capturing process.

\subsection{Object based point cloud alignment}

Several point cloud registration approaches can be found in the literature, however they are often just an extension of the ICP or the NDT [5] process. These iterative methods usually fail if the initial translation is high or the density characteristic is quite different between the target and the source point cloud. Furthermore the typical ring pattern of the Velodyne LIDAR data on the ground region may mislead the registration process by matching the ground region improperly instead of finding correspondence between the foreground regions. So our proposed method, similarly to $[2,8]$ registers the point clouds on the object level to avoid slow and less robust point level transformation estimation.

We divide the $P_{z}$ horizontal plane into $0.2 \mathrm{~m}$ size $2 \mathrm{D}$ grid cells and we assign each point of the point cloud to the corresponding cell. First we mark the ground region based on local neighborhood properties using an adaptive flood fill method starting from the sensor position in the $2 \mathrm{D}$ grid domain [1]. In the next step we extract connected components (objects) by merging the neighboring cells into object candidates (Fig. 3(a), $3(\mathrm{~b}))$.

Since Sec. 3.1 operates with $N=8$ long image sequences, the size of the generated point cloud is much smaller than the ranging distance of the Velodyne sensor, so we only consider an environment with 15 meter radius around the sensor's center.

As a result of the object detection step we extract two sets of object centers $O_{1}$ and $O_{2}$ from the SfMgenerated and the LIDAR-captured point clouds. Our aim is to find the global optimal matching between the two object sets by an iterative voting process [12] in the Hough space. During the transformation estimation we calculate the Euclidean distances between the objects, so finding valid object centers is crucial. Because of the several occlusion and scanning artifacts, larger objects such as facade segments and larger vehicles often fall apart during the object detection. These invalid object centers may mislead the transformation estimation, so first we eliminate larger objects and we only rely on street furniture such as poles, columns, traffic posts and signs, and smaller street objects such as trashes and billboards.

During the transformation estimation we take into account the translation and the rotation component around the upwards vector between the point clouds, because our measurements showed that within the $15 \mathrm{~m}$ search radius the effects of the other two rotation components were negligible. Thus, we define the problem as a $3 \mathrm{D}$ rigid body transformation which can be formulated as a rotation around the upwards vector with the proper $\alpha$ value and a 3D translation among the three coordinate axes. Mathematically we can formulate this transformation as follows:

$$
T_{d x, d y, d z, \alpha}\left(\begin{array}{c}
x \\
y \\
z \\
1
\end{array}\right)=\left[\begin{array}{cccc}
\cos \alpha & \sin \alpha & 0 & d x \\
-\sin \alpha & \cos \alpha & 0 & d y \\
0 & 0 & 1 & d z \\
0 & 0 & 0 & 1
\end{array}\right]\left[\begin{array}{l}
x \\
y \\
z \\
1
\end{array}\right]
$$

Next, we define the transformation estimation as a discrete and finite problem, so we divide the possible transformation space into equal bins. First we allocate a $4 \mathrm{D}$ voting array $V\left[\alpha, d_{x}, d_{y}, d_{z}\right]$ which we can address by the given $\alpha$ rotation value and with the calculated translation components. The algorithm iterates through all the possible $O_{1}$ and $O_{2}$ object center pairs and it rotates $\mathrm{O}_{2}$ with all the possible $\alpha$ values. Thereafter, it calculates the Euclidean distance between the rotated and the other center point:

$$
\left[\begin{array}{l}
d x^{*} \\
d y^{*} \\
d z^{*}
\end{array}\right]=o_{1}-\left[\begin{array}{ccc}
\cos \alpha^{*} & \sin \alpha^{*} & 0 \\
-\sin \alpha^{*} & \cos \alpha^{*} & 0 \\
0 & 0 & 1
\end{array}\right] o_{2}
$$

In each iteration the method increases the evidence of each possible transformation candidate calculated by considering each possible object pair. Finally, we find the maximum value in the voting array which determines the best transformation by the corresponding rotation and translation components and accordingly, we transform the LIDAR point cloud into the coordinate system of the SfM-generated point cloud (Fig. $3(\mathrm{c})$ and $3(\mathrm{~d}))$.

\subsection{Projecting 3D point cloud onto the image domain}

Since we have moved from the LIDAR's space to the SfM-generated point cloud coordinate system using transformation $T_{2}$, we can directly project the points of the LIDAR point cloud onto the $2 \mathrm{D}$ image domain using transformation $T_{1}$. As a result of the projection we can assign the $2 \mathrm{D}$ pixel coordinates to the corresponding $3 \mathrm{D}$ points. Since the absolute position of the LIDAR and the camera sensors is fixed, we transform back the LIDAR point cloud to the original position and based on the 2D-3D mapping we calculate transformation $T_{3}$ which is able to project the $3 \mathrm{D}$ points directly to the image domain without the intermediate transformation $T_{2}$. Knowing the internal parameters of the given camera we can determine transformation $T_{3}$ using the OpenCV solvePnP function which calculates the transformation matrix between a set of $3 \mathrm{D}$ and 2D points using the Levenberg-Marquardt optimization method.

Fig. 4(a) shows the result of the projection of the LIDAR data to the image domain using transformation matrix $T_{3}$. 


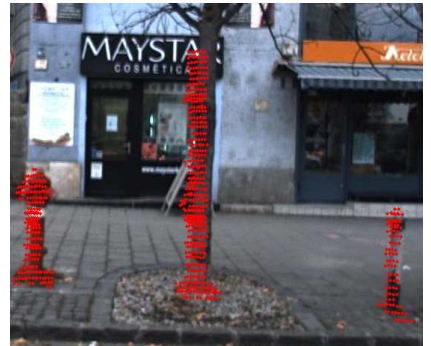

(a) Prop. fully aut. targetless aproach. (seq. 1)

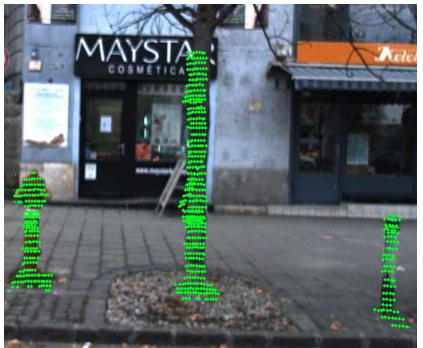

(b) Semi aut. target based [11] approach. (seq. 1)

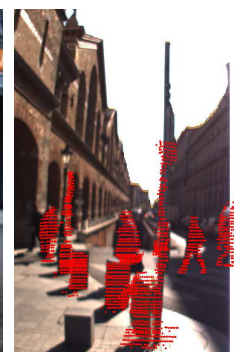

(c) Prop. fully
aproach. (seq. 2)
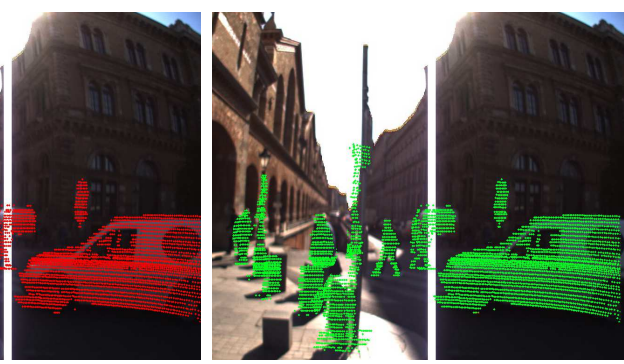

(d) Semi aut. target based [11] approach. (seq. 2)

Figure 4. Qualitative comparison of the proposed online LIDAR-camera self-calibration approach and the [11] state-of-the-art offline calibration algorithm.

\section{Evaluation}

We evaluated our proposed self-calibration method on a new manually annotated dataset (ground truth) and we compared it with a state-of-the-art target based offline calibration [11] method. The database contains two measurement sequences from the streets of an European capital city. While the second sequence was captured in strong sunshine, and the images are suffering from burn-out effect with varying contrast, the first measurement was recorded under cloudy weather conditions resulting in darker images with nearly uniform contrast. We manually annotated 10-10 different images from each sequence, such as heavy main roads, narrow streets and crossroads.

Pixel level projection errors and the standard deviation of the projection errors are shown in Table 1 , furthermore, qualitative comparison versus [11] can be seen in Fig. 4. Although the results show that the offline target-based calibration method can produce higher accuracy registration, calibrating the camera and the LIDAR sensor with [11] method is a lengthy process, taking more than 1 hour, and when parameters change during measurements (e.g., because of sensor displacement) one needs to stop driving and the whole calibration process needs to be repeated.

Another artifact of conventional offline calibration [11] is coming from scanning platform movement: because of the nature of the RMB scanning process, as the speed of the scanning platform is increasing the shape of the point cloud is distorted and it is getting more and more stretched. Since offline calibration can only be performed on a parking vehicle, its accuracy may be decreased as the car moves with higher speed. The effect of this phenomenon is also demonstrated in Fig. 4 the first image pair (Fig. 4(a) and 4(b)) was captured from a static position, and here we can see that the target based reference method slightly outperforms our proposed targetless one. However, by the second pair (Fig. 4(c) and 4(d)), the shot was taken from a quicky moving car and it can be seen that in the current scene the proposed approach overcome the reference one.

The proposed self-calibration method calculates the correspondences between camera and LIDAR online during the operation of the vehicle and calculations can be repeated online periodically, thus, the average $6-7$ pixel error can be considered acceptable considering we process camera images with relatively large resolution $(1288 \times 964)$. At this resolution with $6-7$ pixel error we are able to robustly assign the $3 \mathrm{D}$ objects to the corresponding image regions using the calculated projection matrix, and this data fusion enables the autonomous vehicles to extract more visual features from the surroundings.

There can be situations when the sparse point cloud reconstruction process cannot produce a robust cloud, which might increase registration errors. However, the intended use case of the proposed approach is to periodically repeat the online alignment calculations, and only update the calibration when the current estimation improves the previously used one. Currently we perform such updates at fixed time intervals, however, in the future we also plan to include an automatic step to intelligently find suitable locations based on the current camera images.

Since the proposed self-calibration approach based on an object level alignment method, the quality of the registration is greatly depend on the amount and the type of the detected objects. Our experiments show that the proposed method performs better if the scenes contain column shaped objects such as traffic signs, tree trunks and poles, so after the object detection we count the number of the column shaped objects based on simple geometric constraints and we only calculate the calibration between the camera and the LIDAR if the given scene is appropriate. Typically in the case of main roads and larger crossroads containing several column shaped landmark objects the proposed self-calibration algorithm works more robust and accurate. 


\begin{tabular}{|l|c|c|c|c|}
\hline \multirow{2}{*}{ Error } & \multicolumn{2}{|c|}{ Average x translation error } & \multicolumn{2}{c|}{ Average y translation error } \\
\cline { 2 - 5 } & Average & Deviation & Average & Deviation \\
\hline Semi automatic target based [11] & 2.71 & 0.43 & 3.43 & 0.79 \\
Proposed fully automatic targetless approach & 6.86 & 1.26 & 7.57 & 0.98 \\
\hline
\end{tabular}

Table 1. Performance analysis of the proposed self-calibration approach. Error values are in pixels.

\section{Conclusion}

This paper proposed a targetless camera-LIDAR sensor self-calibration approach using 2D-3D data fusion, that can be performed on the fly, and updated periodically during the data capturing process, thus eliminating the need of lengthy offline sensor calibrations. The method uses a series of camera frames from a short (but continuous) time-window and the captured LIDAR sensor data to perform automatic 2D-3D registration and alignment. We evaluated the proposed method in real life scenarios using real sensors and data.

\section{Acknowledgment}

This work was supported by the National Research, Development and Innovation Fund (grants NKFIA K120233 and KH-125681), and by the Széchenyi 2020 Program (grants EFOP-3.6.2-16-2017-00013 and 3.6.3VEKOP-16-2017-00002). Balázs Nagy was also supported by the ÚNKP-18-3 New National Excellence Program of the Ministry of Human Capacities.

\section{References}

[1] A. Börcs, B. Nagy, and C. Benedek. Fast 3-D urban object detection on streaming point clouds. In Workshop on Computer Vision for Road Scene Understanding and Autonomous Driving at ECCV'14, volume 8926 of $L N C S$, pages 628-639. Springer, Zürich, Switzerland, 2015.

[2] B. Gálai, B. Nagy, and C. Benedek. Crossmodal point cloud registration in the Hough space for mobile laser scanning data. In International Conference on Pattern Recognition (ICPR), pages 3374-3379, Cancun, Mexico, 2016. IEEE.

[3] A. Geiger, F. Moosmann, O. Car, and B. Schuster. Automatic camera and range sensor calibration using a single shot. 2012 IEEE International Conference on Robotics and Automation, pages 3936-3943, 2012.

[4] A. H., B. L.D., and B. B. Automatic calibration of a range sensor and camera system. In 2012 Second International Conference on 3D Imaging, Modeling, Processing, Visualization and Transmission, page pp. 286292, October 2012.

[5] M. Magnusson, A. Nuchter, C. Lorken, A. J. Lilienthal, and J. Hertzberg. Evaluation of $3 \mathrm{D}$ registration reliability and speed - a comparison of ICP and NDT.
In IEEE International Conference on Robotics and Automation, pages 3907-3912, May 2009.

[6] P. Moghadam, M. Bosse, and R. Zlot. Line-based extrinsic calibration of range and image sensors. 2013 IEEE International Conference on Robotics and Automation, pages 3685-3691, 2013.

[7] P. Moulon, P. Monasse, and R. Marlet. Global fusion of relative motions for robust, accurate and scalable structure from motion. In 2013 IEEE International Conference on Computer Vision, pages 32483255, Dec 2013.

[8] B. Nagy and C. Benedek. Real-time point cloud alignment for vehicle localization in a high resolution 3d map. In Workshop on Computer Vision for Road Scene Understanding and Autonomous Driving (ECCV), Germany, 2018.

[9] G. Pandey, J. R. McBride, S. Savarese, and R. M. Eustice. Automatic extrinsic calibration of vision and lidar by maximizing mutual information. Journal of Field Robotics, 32:696-722, 2015.

[10] Y. Park, S. M. Yun, C. S. Won, K. Cho, K. Um, and S. Sim. Calibration between color camera and 3d lidar instruments with a polygonal planar board. In Sensors, 2014.

[11] Z. Pusztai, I. Eichhardt, and L. Hajder. Accurate calibration of multi-lidar-multi-camera systems. In Sensors, 2018.

[12] N. K. Ratha, K. Karu, S. Chen, and A. K. Jain. A realtime matching system for large fingerprint databases. IEEE Transactions on Pattern Analysis and Machine Intelligence, 18(8):799-813, Aug 1996.

[13] S. Rodriguez Florez, V. Fremont, and P. Bonnifait. Extrinsic calibration between a multi-layer lidar and a camera. In 2008 IEEE International Conference on Multisensor Fusion and Integration for Intelligent Systems, pages 214 - 219, 092008.

[14] D. L. Rosenband. Inside waymo's self-driving car: My favorite transistors. In 2017 Symposium on VLSI Circuits, pages C20-C22, June 2017.

[15] D. Scaramuzza, A. Harati, and R. Siegwart. Extrinsic self calibration of a camera and a $3 \mathrm{~d}$ laser range finder from natural scenes. 2007 IEEE/RSJ International Conference on Intelligent Robots and Systems, pages 4164-4169, 2007.

[16] M. Velas, M. Spanel, Z. Materna, and A. Herout. Calibration of rgb camera with velodyne lidar. 2014.

[17] R. Wang, F. P. Ferrie, and J. Macfarlane. Automatic registration of mobile lidar and spherical panoramas. In IEEE Computer Society Conference on Computer Vision and Pattern Recognition Workshops, pages 3340, June 2012. 